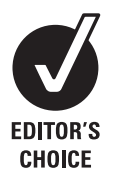

CHOICE

\title{
How to use toxicology screening
}

\section{tests}

\author{
John R H Archer, ${ }^{1,2}$ David M Wood, ${ }^{1-3}$ Paul I Dargan ${ }^{1-3}$
}

\begin{abstract}
${ }^{1}$ Department of Clinical Toxicology, Guy's and St Thomas' NHS Foundation Trust, London, UK

${ }^{2}$ King's Health Partners, London, UK

${ }^{3}$ King's College London, London, UK
\end{abstract}

\section{Correspondence to} Dr John R H Archer, Medical Toxicology Office, 2nd Floor Bermondsey Wing, Great Maze Pond, London SE1 9RT, UK: John.archer@gstt.nhs.uk

Received 30 January 2012 Accepted 21 May 2012 Published Online First 21 July 2012

\section{Introduction}

The evaluation of poisoned patients presenting to the hospital predominantly relies on clinical assessment based on the history of ingestion/exposure and clinical symptoms/signs. ${ }^{1}$ The role of toxicological screening is controversial. In the emergency department (ED), a toxicology screen (TS) can be used to detect a substance that causes the clinical features in an individual. Therefore, as a diagnostic test, the term 'screen' can be misleading. A toxicology screen generally involves qualitative detection of specific drugs or metabolites in biological specimens. Detection indicates that a substance is present; however, it does not indicate whether a patient's symptoms/signs are caused by the presence of this substance, and it is important to exclude other causes. Therefore, a toxicological screen performs poorly as a diagnostic test. ${ }^{2}$

In addition to a general TS, there are specific drug/chemical assays that can be used to guide clinical management of poisoned patients (see box 1). ${ }^{3}$ These are used to measure the concentration (or 'level') of a drug/chemical, and are the most useful type of screening used to manage a poisoned patient. The poisonings for which these are useful are those in which the serum concentration of the drug/chemical correlates to the clinical effects and toxicity, and will either determine treatment (eg, $\mathrm{N}$-acetylcysteine treatment in paracetamol poisoning) or help guide it (eg, urinary alkalinisation in salicylate poisoning) (box 1). These tests are ordered selectively when particular drug exposures are suspected, either on the basis of the history, or through characteristic clinical features.

The decision to use a toxicological screening test depends on clinical features, the suspected substances involved and the influence it may have on patient management. This paper presents the evidence for the role of toxicological screening in paediatric practice.

\section{Physiological background: what is a toxicology screen, and how is it conducted?}

A TS involves multiple tests performed on one or more biological specimens to determine whether drugs/chemicals are present. The extent of the screen and the number of drugs/chemicals included depends on the laboratory. Generally, the screen tests for common pharmaceuticals and/or several commonly abused recreational drugs (and/or their metabolites) including opioids, cocaine, cannabis, amphetamines and benzodiazepines. Hospital laboratories vary in their capabilities, some more able to perform assays for numerous drugs on biological specimens, including pharmaceuticals, recreational drugs and chemicals, such as toxic alcohols (eg, ethylene glycol, methanol) and novel recreational drugs.

\section{Suitable biological specimens in paediatrics}

Testing for drugs in different biological specimens offers many advantages, the most important of which is objectivity. In children, a variety of samples can be used, including venous blood, umbilical cord blood, urine, hair and meconium. Both venous and cord blood specimens will yield quantitative results giving a concentration of a drug - this is most useful for substances with a low volume of distribution for which the blood concentration correlates with overall body burden. Urine specimens supply qualitative data (presence or absence) and are suited for detecting drugs with a large volume of distribution and for confirmation of exposure. Although quantitative analysis can be undertaken on urine specimens, these cannot be used to guide treatment and generally, therefore, qualitative analysis, for example, an immunoassay, is used. These tests have good negative predictive value; however, positive results need to be interpreted with caution. Positive screening has a high sensitivity, but can lack 
Box 1 Quantitative drug assays that determine or guide treatment

Drug concentrations that determine treatment Paracetamol

Drug/chemical concentrations that guide treatment Anticonvulsants (carbamazepine, phenytoin, valproate) Aspirin

Carbon monoxide

Digoxin

Ethanol

Ethylene glycol

Iron

Methaemoglobin

Lead

Lithium

Methanol

Theophylline

specificity due to the inherent technical difficulties that are common with immunoassays, for example, interference by other pharmaceuticals, cross-reactivity with drug metabolites and with structurally similar substances (over-the-counter cold preparations cause positive results for amphetamines). It is therefore important that initial screening results are confirmed by further specific testing using a technique such as gas chromatography with mass spectrometry. This confirmatory analysis is labour intensive, requires specific skills, is expensive and time consuming. Therefore and because, generally, the management of patients with recreational drug toxicity can be guided by clinical features, confirmatory analyses are not routinely undertaken in either adults or children with acute recreational drug toxicity.

Although blood and urine are typically used for toxicological screening, the presence of drugs in these fluids is generally short-lived. The time limit for detection varies, but is generally less than $24 \mathrm{~h}$ for parent drug, although metabolites may be detectable over longer periods. Therefore, detection becomes limited by time from exposure, and the use of alternative specimens may be more appropriate if confirmation of drug use is required at a later stage. ${ }^{4}$ This is especially true for newborns whose mothers have abused drugs during their pregnancy. In such instances, neonatal hair can be used to assess chronic drug exposure. Its advantages include ease of collection, specimen stability and a protracted window of drug detection..$^{5}$ At the time of hair follicle formation, drugs can be incorporated into the hair shaft and remain in the shaft indefinitely, and can therefore be subjected to analysis. ${ }^{6}$ Hair grows at a rate of approximately $1 \mathrm{~cm} /$ month, therefore, analysis of sequential segments can provide an estimate of drug exposure over time. ${ }^{6}$ Neonatal hair begins to form at approximately 20 weeks of gestation, and like adult hair, will incorporate drugs present in the foetal circulation. ${ }^{7} 8$
A limitation of adult hair analysis is the possibility of hair surface contamination from the environment which is not an issue in testing a newborn's hair if sampling takes place immediately postpartum and prior to discharge from hospital.

Toxicological testing may also be performed on meconium. Meconium begins to form at approximately 12 weeks of gestation on the initiation of foetal swallowing. ${ }^{9}$ The mechanism by which drugs are deposited into meconium is not clear. When a foetus is exposed to drugs through maternal consumption, it excretes them into bile/foetal urine into the amniotic fluid. Drugs are secreted into meconium by deposition from bile or swallowing of amniotic fluid. ${ }^{10}$ As meconium is not excreted in utero, it can be used as an estimate of in utero drug exposure as it is considered to be static once deposited in the foetal intestine. ${ }^{11}$ Therefore, it can provide a longer retrospective view of foetal exposure than that afforded by blood, urine and even the newborn's hair. ${ }^{10}$

\section{What procedures should be instigated once biological samples have been taken from a suspected case of deliberate paediatric poisoning?}

The documentation of the presence of an illicit drug in a newborn, infant or adolescent can be challenged by the mother or family. Furthermore, reporting of positive findings to local authorities may result in issues relating to child custody, therefore, special care in performing and reporting tests is required. This may require a chain-of-custody documentation which provides proof that the specimen has been maintained appropriately and not been inappropriately interfered with. Details relating to those who handled the specimen, right from the specimen collector to the person responsible for the specimen disposal, should be recorded. The time and reason for each change in custody (ie, transfer from person to person) should be recorded. Aliquoting of the sample needs to be fully accounted for, and laboratory areas where processing, analysis and storage are involved, should be secure with restricted access only to those appropriately authorised individuals.

\section{Clinical indications}

Is a toxicology screen indicated in every newborn has signs or symptoms compatible with substance abuse?

The impact of prenatal drug abuse on neonatal health and development has been well documented and remains a major health concern. ${ }^{12-14}$ Rates of prenatal drug use determined by maternal reports and biological specimen analysis range from $3.4-31 \%$ for cocaine, $1-12 \%$ for cannabis, $1.6-21 \%$ for opiates and $0.1-4 \%$ for ethanol in general populations and those with a history of current/previous drug use. ${ }^{15}$ In the short term, newborns exposed to substance abuse during 
Box 2 Example guideline for identifying at-risk newborns of prenatal drug exposure ${ }^{22}$

Indications for newborn toxicology screening for prenatal drug exposure

Maternal factors

Infants whose mothers have any of the following:

A. History of drug abuse in present or previous pregnancies

B. Limited/no prenatal care

C. History of hepatitis B, AIDS, syphilis, gonorrhoea, prostitution

D. Unexplained placental abruption

E. Unexplained premature labour

Newborn factors

Infants who have any of the following:

A. Unexplained neurological complications, for example, intracranial haemorrhage/infarction, seizures

B. Evidence of possible drug withdrawal, for example, hypertonia, irritability, seizures, tremulous, muscle rigidity, decreased or increased drooling

C. Unexplained intrauterine growth retardation

pregnancy may develop central nervous system irritability, decreased growth parameters and severe withdrawal symptoms. ${ }^{13}{ }^{16-18}$ In the long term, effects include sudden infant death syndrome, abnormal neurocognitive/behavioural development and child abuse/ neglect. ${ }^{19-21}$ Infants at high risk of substance exposure (box 2), therefore, need to be reliably identified (and followed up) when suggestive clinical features are present, such as unexplained neurological symptoms and/or symptoms of possible drug withdrawal. Previously, research groups have developed guidelines that identify maternal and newborn factors associated with substance abuse (box 2). Tested prospectively, these have demonstrated $89 \%$ sensitivity in confirming the association of specific maternal characteristics with maternal cocaine use. ${ }^{22} 23$ Toxicological screening performed in this way removes the bias in physician-ordered tests, therefore protecting their clinical decision. It reduces costs by avoiding universal screening and allows improved identification of newborns exposed to prenatal substance abuse.

\section{Does a negative urine toxicology screen from at-risk newborns exclude maternal substance abuse during pregnancy?}

Drug testing is one of the only methods of definitively identifying in utero drug exposure, as reliance on maternal history of drug use may prove unreliable. ${ }^{24}$ Toxicological screening on neonatal blood and urine has a limited window of detection and reflects only foetal drug exposure during the previous hours or days before collection, and not chronic exposure. At times, sufficient neonatal plasma can be difficult to collect, and other matrices, for example, urine, is preferred. ${ }^{25}$ Although less invasive, the drawback of urine collection is that drugs clear rapidly from urine, and so the time required for successful collection is critical. ${ }^{26}$ False negatives are therefore possible, and so a negative urine screen cannot definitely rule out maternal prenatal substance abuse. ${ }^{5}$ Furthermore, there are no recommended thresholds for testing clinical samples in laboratories. Adopted thresholds used in workplace drug testing may be too high versus the lowest detectable limits, contributing further to false negatives.

Toxicology screening for chronic drug use during pregnancy can be carried out on high-risk newborns by using meconium, or hair. ${ }^{14}$ For detecting first-time drug use by the mother just prior to delivery, meconium may give a false negative if the drug tested for has not had time to deposit in the meconium. In these cases, screening requires both urine and meconium analysis to cover potential time periods from drug exposure. Compared with meconium, hair can provide a chronological breakdown of drug exposure with the ability to differentiate between single/occasional and repeated drug exposure. However, the analysis of a newborn's hair is technically demanding and, therefore, only available in a few specialised laboratories.

Will the use of intrapartum opioids influence a newborn's urine toxicology screen?

Analgesics, such as opioids, are often used during labour and the immediate post-delivery phase for medical management of maternal pain, neonatal pain after delivery and maternal chronic medical conditions. Opioids can be divided into distinct groups; naturally occurring (morphine, codeine), semi-synthetic (heroin, oxycodone, pethidine) and synthetic opioids (fentanyl, methadone, tramadol). Synthetic opioids show little or no cross-reactivity in routine opiate immunoassays. Semi-synthetic opioids derived from morphine will show variable cross-reactivity, the extent of which depends on the manufacturer of the immunoassay. Clinicians need to be aware of the poor detection of these opioids, and that a positive test may not relate to the medical use of opioids during or around the time of labour. Additional tests would be required to exclude opioids used in pain management during labour.

\section{Is toxicology screening of value in young children admitted to the emergency department with an apparent life-threatening event?}

Children are exposed to toxic substances more frequently than any other age group. ${ }^{27}$ Annual statistics taken from the American Association of Poison Control Centres for 2009-2010 show that 51.9\% of total paediatric exposures (approximately 1.6 million) were in children $<6$ years old, and $38.9 \%$ in children $\leq 2$ years old. ${ }^{27}$ The majority are unintentional, small ingestions that do not result in significant clinical features. However, a small minority are potentially life threatening. From the same data, $6.8 \%$ of total deaths (1158 deaths) occurred in paediatric patients, with 
$1.8 \%$ occurring in those <6 years old, and $1.4 \%$ occurring in those $\leq 2$ years old. ${ }^{27}$

It is, therefore, important to consider a toxicological aetiology in infants with unexplained symptoms/ changes in behaviour as an apparent life-threatening event (ALTE). The diagnosis of an ALTE is often not immediately evident; however, poisoning, intentional or unintentional, has been described as a cause in a number of cases. ${ }^{28}$ In a systematic study of 274 children $<2$ years old presenting to the ED with an ALTE, a comprehensive urine TS was completed. ${ }^{29}$ Results of $8.4 \%$ were found to be positive with clinical findings that were thought to represent a possible cause. Furthermore, $4.7 \%$ of screen results were positive for an over-the-counter cough and cold preparation; however, the finding was not compatible with the respective drug history and was not admitted to being given by the child's carer. While such poisoning in young children may inadvertently occur through breastfeeding, it may also be more overt during misguided attempts to treat the symptoms of a viral or other non-specific illness. Furthermore, the possibility of deliberate harm as part of a malicious exposure by carers should also be considered. Although there are no consensus guidelines supporting the routine use of TSs in ALTEs, the authors of this study suggest that it be part of their routine evaluation.

\section{Can a toxicology screen alter hospital management in older children and adolescents suspected of poisoning?} Drug exposures in older children, and in particular adolescents, are similar to those in adults, and often involve intentional ingestion of medications and recreational drugs. ${ }^{30}$ These are generally associated with higher rates of morbidity/mortality than the exposures in younger children. In 2009 in the USA, $6.2 \%$ of all paediatric drug exposures occurred between the ages of 13 and 19 years, with $4.2 \%$ of all mortality from drug exposure occurring in this group-an increase of 2.3 times compared with children $<6$ years. ${ }^{27}$ Despite this, toxicology screening in this age group seldom influences management. In a retrospective study of 338 paediatric patients $(77 \%>12$ years old) in whom a TS had been performed in the ED as part of routine clinical assessment, $22(7 \%)$ were found to have an unexpected positive result. ${ }^{31}$ Samples were analysed by gas chromatography with mass spectrometry, but the specific drugs/chemicals screened for were not described. Based on the unexpected results, three patients $(1 \%)$ had an alteration in medical management (admission to hospital). In these cases, each child had an abnormal physical examination and vital signs that, retrospectively, were consistent with the drug ingested, including two with tricyclic antidepressant toxicity and one with Munchausen's syndrome, by proxy, involving phenobartal/carbamezepine toxicity. The authors concluded that toxicology screening rarely alters medical management, and that emergency physicians should re-evaluate the indications for such testing in the paediatric population. ${ }^{31}$

In a further retrospective study, 463 comprehensive TSs from paediatric patients (mean \pm SD age $11 \pm 6$ years) incorporating $>550$ potential toxins were reviewed. ${ }^{32}$ Of 234 positive tests $(51 \%)$ for one or more toxins, 7 (3\%) occurred in cases in which there was no documented suspicion of an exposure. However, none resulted in a change in medical management. Furthermore, the estimated cost of the analysis ( $\$ 2315$ per patient) appeared excessive in relation to the lack of impact on clinical management or outcome. In another study, treating physicians were prospectively asked to document whether TS affected a patient's management using a utility rating scale pre- and post-screening. ${ }^{33}$ In total, 158 TSs from paediatric patients were requested due to a history of ingestion or altered mental status. A total of $90 \%$ of children $>12$ years old had TS compared with $60 \%<6$ years $(\mathrm{p}<0.001)$. In total, $78(49 \%)$ screens were positive, and in $53(34 \%)$ cases the treating physician stated that this result impacted on patient management. Of these, significant differences in pre- and post-test utility values were found for serum assays $(p=0.008)$, altered mental status $(p=0.005)$ and patients with a negative drug test $(p=0.003)$. Of 17 unexpected findings, only 4 ( $3 \%$ of total) influenced patient management. The study concluded that toxicology screening is influential on patient management when it involved quantitative serum assays, for example, paracetamol, aspirin, but not qualitative toxicology screening. ${ }^{33}$

\section{What are the main limitations of toxicology screening?}

Negative toxicology screens

TS can provide physicians with 'objective' reassurance; however, this is generally unhelpful clinically because of the limited capability of most screening tests and the time frame for which drugs (and/or their metabolites) remain detectable. A negative qualitative TS cannot entirely exclude exposure to a compound, but conclude that the compound, at the time of testing, was not present at the minimum threshold quantity. In such cases, a physician may be misled by a negative result interpreting it as meaning 'drug not present' when in fact it only means 'drug not detected'. ${ }^{34}$ When interpreting a negative TS, a physician must be aware of the extent of the screen used both in terms of the drugs/chemicals screened for and the sensitivity/limit of detection.

\section{Positive toxicology screens}

Specific quantitative serum assays have a greater impact on patient management than a positive qualitative toxicology screening assay. However, defining the severity of intoxication and/or guiding specific treatment based purely on the concentration of a drug/ chemical in a blood sample is not always possible. While qualitative assays are extremely sensitive, they 
lack specificity and are at risk of false positives. As such, a positive result does not confirm that a drug is present in a clinically significant quantity. It may also be misinterpreted, as drug metabolites and many other substances can cause interference and cross-react with drug-of-abuse assays for many days following exposure. ${ }^{1}$ Furthermore, while a positive result may help explain a clinical presentation, consideration and exclusion of other treatable causes are required. A TS is designed as an adjunct to facilitate a diagnosis and must be interpreted in the context of both, the clinical features present and other indicators, such as acid-base and other biochemical features.

\section{Future research}

Toxicology screening using urine and/or blood samples can be effective in facilitating a diagnosis in children. However, in neonates, it remains unclear as to which biological specimens are best to evaluate in utero exposure, or indeed whether monitoring pregnant women who are suspected of illicit drug use can be linked to observed neonatal or later developmental outcomes. To effectively assess the effects of illicit drugs on a foetus, further research is required into maternal monitoring to establish when monitoring should commence, the frequency of monitoring, whether quantitative drug concentrations are helpful and which sampling method is most suitable. Blood and urine have been the preferred biological samples for drug testing; however, non-invasive methods, such as hair, sweat and oral fluid are now possibilities that require further validation in this setting.

\section{Clinical bottom line}

Paediatric toxic exposures are common, and while a few do result in significant morbidity/mortality, they are often treatable. Physicians using a combined assessment of history and clinical examination are often able to predict substance abuse, ${ }^{35}$ although histories from patients (or their carers) suspected of drug ingestion may be unreliable. Data on the use and clinical utility of comprehensive drug testing in children is sparse. Hospital laboratories vary in the comprehensiveness of the toxicology screening that they can perform. Clinicians ordering a TS should, therefore, ensure that their request is tailored to the clinical presentation and the type of poison suspected. Laboratories offering comprehensive toxicology screens can add to the clinical decision making for patients suspected of drug overdose. This provides reassurance and improves agreement among physicians on patient outcome and, therefore, potentially saving resources. ${ }^{33} 3637$ However, routine qualitative toxicology screening, often for drugs of abuse, has been shown to rarely influence overall clinical management. ${ }^{31-33}$ Specific quantitative screening for specific poisons, for example, paracetamol, aspirin, lithium (box 1) assists in patient management, and is probably the most helpful of available toxicology tests.

A rational and pragmatic approach to toxicology screening should be applied in paediatric care where its utility differs across different age groups. On the basis of the literature reviewed in this paper, we believe that toxicology screening may be of benefit in the following situations:

1. In neonates, to confirm drug exposures for forensic purposes, such as assessing for in utero exposure to illicit drugs or detecting malicious drug exposure.

2. In children, to screen for illicit drugs, where the presenting history is questionable, or unexplained symptoms are present (eg, coma, altered behaviour), and ingestion is in the differential diagnosis and/or malicious drug exposure is suspected.

3. In children and adolescents, when a specific therapy might be initiated based on a high serum level, for example in paracetamol or aspirin ingestion.

\section{Competing interests None.}

Provenance and peer review Commissioned; externally peer reviewed.

\section{References}

1. Greene SL, Dargan DI, Jones AL. Acute poisoning: understanding $90 \%$ of cases in a nutshell. Postgrad Med J $2005 ; 81: 204-16$.

2. Sackett DL, Holland WW. Controversy in the detection of disease. Lancet 1975;2:357-9.

3. National Poisons Information Service, Association of Clinical Biochemists. Laboratory analyses for poisoned patients: joint position paper. Ann Clin Biochem 2002;39:328-39.

4. Compton P. The role of urine toxicology in chronic opioid analgesic therapy. Pain Manag Nurs 2007;8:166-72.

5. Lozano J, García-Algar O, Vall O, et al. Biological matrices for the evaluation of in utero exposure to drugs of abuse. Ther Drug Monit 2007;29:711-34.

6. Pragst F, Balikova MA. State of the art in hair analysis for detection of drug and alcohol abuse. Clin Chim Acta 2006;370:17-49.

7. Akiyama M, Matsuo I, Shimizu H. Formation of cornified cell envelope in human hair follicle development. $\mathrm{Br} J$ Dermatol 2002;146:968-76.

8. Bar-Oz B, Klein J, Karaskov T, et al. Comparison of meconium and neonatal hair analysis for detection of gestational exposure to drugs of abuse. Arch Dis Child Fetal Neonatal Ed 2003;88:F98-100.

9. Koren G, Hutson J, Gareri J. Novel methods for the detection of drug and alcohol exposure during pregnancy: implications for maternal and child health. Clin Pharmacol Ther 2008;83:631-4.

10. Ostrea EM Jr, Brady MJ, Parks PM, et al. Drug screening of meconium in infants of drug-dependent mothers: an alternative to urine testing. J Pediatr 1989;115:474-7.

11. Abusada GM, Abukhalaf IK, Alford DD. Solid-phase extraction and GC/MS quantitation of cocaine, ecgonine methyl ester, benzoylecgonine, and cocaethylene from meconium, whole blood, and plasma. J Anal Toxicol 1993; 17:353-8. 
12. Ostrea EM, Chavez CJ. Perinatal problems (excluding neonatal withdrawal) in maternal drug addiction: a study of 830 cases. J Pediatr 1979;94:292-5.

13. Bell GL, Lau K. Perinatal and neonatal issues of substance abuse. Pediatr Clin North Am 1995;42:261-81.

14. Ostrea EM Jr, Brady M, Gause S, et al. Drug screening of newborns by meconium analysis: a large-scale, prospective, epidemiologic study. Pediatrics 1992;89:107-13.

15. Gareri J, Klein J, Koren G. Drugs of abuse testing in meconium. Clin Chim Acta 2006;366:101-11.

16. Chasnoff IJ, Griffith DR. Cocaine: clinical studies of pregnancy and the newborn. Ann N Y Acad Sci 1989;562:260-6.

17. Dixon SD. Effects of transplacental exposure to cocaine and methamphetamine on the neonate. West J Med 1989;150:436-42.

18. Franck L, Vilardi J. Assessment and management of opioid withdrawal in ill neonates. Neonatal Netw 1995;14:39-48.

19. Kandall SR, Gaines J, Habel L, et al. Relationship of maternal substance abuse to subsequent sudden infant death syndrome in offspring. J Pediatr 1993;123:120-6.

20. van Baar AL, Soepatmi S, Gunning WB, et al. Development after prenatal exposure to cocaine, heroin and methadone. Acta Paediatr Suppl 1994;404:40-6.

21. Jaudes PK, Ekwo E, Van Voorhis J. Association of drug abuse and child abuse. Child Abuse Negl 1995;19:1065-75.

22. Ryan RM, Wagner CL, Schultz JM, et al. Meconium analysis for improved identification of infants exposed to cocaine in utero. J Pediatr 1994;125:435-40.

23. Farst KJ. Newborns exposed to illicit drugs in-utero. Antenatal and Neonatal Guidelines, University of Arkansas for Medical Sciences 2009. http://www.uams.edu/ANGELS/

24. Ostrea EM Jr, Knapp DK, Tannenbaum L, et al. Estimates of illicit drug use during pregnancy by maternal interview, hair analysis, and meconium analysis. J Pediatr 2001;138:344-8.

25. Eyler FD, Behnke M, Wobie K, et al. Relative ability of biologic specimens and interviews to detect prenatal cocaine use. Neurotoxicol Teratol 2005;27:677-87.
26. Halstead AC, Godolphin W, Lockitch G, et al. Timing of specimen collection is crucial in urine screening of drug dependent mothers and newborns. Clin Biochem 1988;21:59-61.

27. Bronstein AC, Spyker DA, Cantilena LR Jr, et al. 2009 Annual Report of the American Association of Poison Control Centers' National Poison Data System (NPDS): 27th Annual Report. Clin Toxicol (Phila) 2010;48:979-1178.

28. Hickson GB, Altemeier WA, Martin ED, et al. Parental administration of chemical agents: a cause of apparent life-threatening events. Pediatrics 1989;83:772-6.

29. Pitetti RD, Whitman E, Zaylor A. Accidental and nonaccidental poisonings as a cause of apparent life-threatening events in infants. Pediatrics 2008;122:e359-62.

30. Gauvin F, Bailey B, Bratton SL. Hospitalizations for pediatric intoxication in Washington State, 1987-1997. Arch Pediatr Adolesc Med 2001;155:1105-10.

31. Sugarman JM, Rodgers GC, Paul RI. Utility of toxicology screening in a pediatric emergency department. Pediatr Emerg Care 1997;13:194-7.

32. Belson MG, Simon HK. Utility of comprehensive toxicologic screens in children. Am J Emerg Med 1999;17:221-4.

33. Belson MG, Simon HK, Sullivan K, et al. The utility of toxicologic analysis in children with suspected ingestions. Pediatr Emerg Care 1999;15:383-7.

34. Wiley JF. Difficult diagnoses in toxicology. Poisons not detected by the comprehensive drug screen. Pediatr Clin North Am 1991;38:725-37.

35. Kozer E, Bar-Hamburger R, Rosenfeld NY, et al. Illicit drug and alcohol users admitted to the pediatric emergency department. Isr Med Assoc J 2008;10:779-82.

36. Fabbri A, Marchesini G, Morselli-Labate AM, et al. Comprehensive drug screening in decision making of patients attending the emergency department for suspected drug overdose. Emerg Med J 2003;20:25-8.

37. Moeller KE, Lee KC, Kissack JC. Urine drug screening: practical guide for clinicians. Mayo Clin Proc 2008;83:66-76. 